\title{
Neuroinflammation in fibromyalgia and CRPS is multifactorial
}

\section{Alex Vasquez}

In his Review article (Neurogenic neuroinflammation in fibromyalgia and complex regional pain syndrome. Nat. Rev. Rheumatol. 11, 639-648; 2015) ${ }^{1}$, Geoffrey Littlejohn ascribes neuroinflammation to a "neurogenic" origin, presumably triggered by pain and stress. However, attribution of neuroinflammation and central sensitization to a primary neurogenic origin is premature without integrating the well-documented coexistence of small intestine bacterial overgrowth (SIBO, one type of gastrointestinal dysbiosis), vitamin D deficiency, and mitochondrial dysfunction.

Littlejohn ${ }^{1}$ notes that chronic pain has been associated with lipopolysaccharide (LPS)-stimulated proinflammatory cytokines (particularly IFN- $\gamma$ and TNF); however, he does not pursue this line of thought to connect it to relevant literature showing clear evidence of gastrointestinal dysbiosis and increased intestinal permeability in patients with fibromyalgia and complex regional pain syndrome (CRPS). The gastrointestinal tract is the most abundant source of LPS, systemic absorption of which is increased by SIBO and increased intestinal permeability. In 1999, Pimentel et al. ${ }^{2}$ showed that oral administration of antibiotics led to alleviation of pain and other clinical measures of fibromyalgia. In 2004, Pimentel et al. ${ }^{3}$ showed that among 42 fibromyalgia patients, all (100\%) showed laboratory evidence of SIBO, severity of which correlated positively with severity of fibromalgia. In that same year, Wallace and Hallegua ${ }^{4}$ showed that eradication of SIBO with antimicrobial therapy led to clinical improvements in fibromyalgia patients in direct proportion to antimicrobial efficacy. In 2008, Goebel et al. ${ }^{5}$ documented that patients with fibromyalgia and CRPS have intestinal hyperpermeability; mucosal "leakiness" was highest in patients with CRPS, indicating a strong gastrointestinal component to the illness. In 2013, Reichenberger et al. ${ }^{6}$ showed that CRPS patients have a distinct alteration in their gastrointestinal microbiome characterized by reduced diversity and significantly increased levels of Proteobacteria. LPS from Gram-negative bacteria is powerfully proinflammatory and is known to trigger microglial activation via Toll-like receptor 4; experimental studies have shown that LPS promotes muscle mitochondrial impairment, peripheral hyperalgesia, and central sensitization ${ }^{7}$.

Vitamin D deficiency is prevalent in chronic pain and fibr+omyalgia patients and promotes pain sensitization, myalgia and bone pain (osteomalacia) ${ }^{8}$. Human clinical trials have shown that vitamin D supplementation can alleviate inflammation ${ }^{9}$, intestinal hyperpermeability ${ }^{10}$, fibromyalgia pain ${ }^{11}$ and other neuromusculoskeletal pain. Vitamin D reduces experimental microglial activation ${ }^{12}$, a component of neuroinflammation and central sensitization.

Mitochondrial dysfunction, noted in fibromyalgia ${ }^{13}$ and $\mathrm{CRPS}^{14}$, may be triggered by gastrointestinal dysbiosis via LPS, D-lactate, hydrogen sulfide, and inflammation; mitochondrial dysfunction exacerbates and perpetuates microglial activation and glutaminergic neurotransmission ${ }^{15}$, thereby promoting pain sensitization centrally while also contributing to muscle pain peripherally ${ }^{7}$. Treatment of mitochondrial dysfunction with ubiquinone alleviates many biochemical and clinical manifestations of fibromyalgia ${ }^{13}$.

Thus, neuroinflammation in fibromyalgia and CRPS has biological contributions including gastrointestinal dysbiosis, vitamin D deficiency, and mitochondrial dysfunction. These independent contributions commonly coexist, and each of these is additive/synergistic with the others in the promotion of peripheral and central hyperalgesia. The consistent pain-alleviating benefits of treatments for intestinal dysbiosis (antibiotics), vitamin D deficiency (supplementation) and mitochondrial dysfunction (ubiquinone) establish that these painful conditions are multifactorial and maintained by ongoing physiologic insults, each of which is treatable.

Alex Vasquez is at the International College of Human Nutrition and Functional Medicine, Calle Balmes 184, $3^{\circ} 3^{a}$, Barcelona, Spain 08006 avasquez@ichnfm.org

doi:10.1038/nrrheum.2016.25 Published online 3 Mar 2016

1. Littlejohn, G. Neurogenic neuroinflammation in fibromyalgia and complex regional pain syndrome. Nat. Rev. Rheumatol. 11, 639-648 (2015).

2. Pimentel, M. et al. Improvement of symptoms by eradication of small intestinal overgrowth in FMS: a double-blind study [abstract]. Arthritis Rheum. 42, S343 (1999)

3. Pimentel, M. et al. A link between irritable bowel syndrome and fibromyalgia may be related to findings on lactulose breath testing. Ann. Rheum. Dis. 63 , 450-452 (2004).

4. Wallace, D. J. \& Hallegua, D. S. Fibromyalgia: the gastrointestinal link. Curr. Pain Headache Rep. 8, 364-368 (2004)

5. Goebel, A., Buhner, S., Schedel, R., Lochs, H. $\&$ Sprotte, G. Altered intestinal permeability in patients with primary fibromyalgia and in patient with complex regional pain syndrome. Rheumatology 47, 1223-1227 (2008).

6. Reichenberger, E. R. et al. Establishing a relationship between bacteria in the human gut and complex regional pain syndrome. Brain Behav. Immun. 29 62-69 (2013)

7. Vasquez, A. Human Microbiome and Dysbiosis in Clinical Disease 2015 (International College of Human Nutrition and Functional Medicine, 2015).

8. von Känel, R., Müller-Hartmannsgruber, V. Kokinogenis, G. \& Egloff, N. Vitamin D and central hypersensitivity in patients with chronic pain. Pain Med. 15, 1609-1618 (2014).

9. Timms, P. M. et al. Circulating MMP9, vitamin D and variation in the TIMP-1 response with VDR genotype: mechanisms for inflammatory damage in chronic disorders? OJM 95, 787-796 (2002).

10. Raftery, T. et al. Effects of vitamin D supplementation on intestinal permeability, cathelicidin and disease markers in Crohn's disease: results from a randomised double-blind placebo-controlled study. United European Gastroenterol. J. 3, 294-302 (2015).

11. Wepner, F. et al. Effects of vitamin D on patients with fibromyalgia syndrome: a randomized placebocontrolled trial. Pain 155, 261-268 (2014).

12. Hur, J., Lee, P., Kim, M. J. \& Cho, Y. W. Regulatory effect of 25-hydroxyvitamin $D_{3}$ on nitric oxide production in activated microglia. Korean J. Physiol. Pharmacol. 18, 397-402 (2014).

13. Cordero, M. D. et al. Oxidative stress correlates with headache symptoms in fibromyalgia: coenzyme $\mathrm{Q}_{10}$ effect on clinical improvement. PLoS One 7, e35677 (2012).

14. Tan, E. C. et al. Mitochondrial dysfunction in muscle tissue of complex regional pain syndrome type I patients. Eur. J. Pain 15, 708-715 (2011).

15. Nguyen, D. et al. A new vicious cycle involving glutamate excitotoxicity, oxidative stress and mitochondrial dynamics. Cell Death Dis. 8, e240 (2011).

\section{Competing interests statement}

The author declares that he has worked as a consultant for Biotics Research Corporation (a nutraceutical company based in the USA), and that he has lectured and written for this company on various topics, including fibromyalgia. 